\title{
Explaining Inconsistency of AHP-comparison Matrix by Decomposition Analysis
}

\author{
Pertti Laininen \\ Helsinki University of Technology, Systems Analysis Laboratory, P.O.Box 1100, Fin-02015 Hut, \\ Finland, Telefax: +358-9-4513096. E-mail: Pertti.Laininen@hut.fi
}

\begin{abstract}
The standard method to estimate the values for the weights of criteria or decision alternatives in the analytic hierarchy process (AHP) is to make pairwise comparisons of the objects by using Saaty's comparison scale, where the comparison ratios are formed with numbers $1 / 9,1 / 8, \ldots, 8,9$. The values of the weights are the normalized components of the principal eigenvector associated to the largest eigenvalue of the comparison matrix. In practice, the comparisons are inconsistent to some extent, and therefore the eigenvalue method to calculate the weights becomes problematic. Saaty has studied the effect of inconsistency on the eigenvector solution, and he gives an advice not to use the method if the consistency exceeds some measure. More precisely, the so-called consistency ratio should not exceed the value 0.10 (Saaty[2]).

Gass and Rapcsák [1] present an application of the singular value decomposition (SVD) for the comparison matrix in order to obtain better estimates for the weights. They prove that the rank one left and right singular vectors, which are connected to the largest singular value, yield theoretically welljustified weights. In an inconsistent case this approach seems to be more reliable than the usual eigenvector method. Unfortunately, there is a drawback in the use of SVD to the basic comparison matrix. The comparisons are done in ratio scale, but SVD is a linear transformation that spans in a linear way the distances between the alternatives (the distances between the columns or between the rows of the comparison matrix).
\end{abstract}

The main features of the decomposition analysis (DA) are the following.

Let $\mathbf{R}$ be a positive reciprocal AHP-comparison matrix with size $n \times n$. By taking logarithm of the elements of $\mathbf{R}$ we get a skew symmetric matrix $\mathbf{L}=\log (\mathbf{R})$ with the signed distances between the alternatives as its elements. In this matrix the comparison ratios have been transformed linear. Singular value decomposition of $\mathbf{L}$ is a product of three matrices, $\mathbf{L}=\mathbf{U S V}$ ' where $\mathbf{S}$ is diagonal matrix of singular values, $\mathbf{S}=\operatorname{diag}\left(\sqrt{\delta_{1}}, \sqrt{\delta_{1}}, \ldots, \sqrt{\delta_{k}}, \sqrt{\delta_{k}}, 0, \ldots, 0\right)$ for some $2 \mathrm{k} \leq \mathrm{n}$. $\mathbf{U}$ and $\mathbf{V}$ are matrices of the eigenvectors connected to the eigenvalues of the matrices $\mathbf{L} \mathbf{L}$ ' and $\mathbf{L}$ ' $\mathbf{L}$. Here $\delta_{1}$ is the largest eigenvalue of $\mathbf{L L}$ ' (there are two equal eigenvalues $\boldsymbol{\delta}_{1}$ ), and $\boldsymbol{\delta}_{k}$ is the k:th eigenvalue of $\mathbf{L L}$ ' (there are two equal eigenvalues $\delta_{k}$ ). The smallest eigenvalues are zeros. If $\mathbf{R}$ is a consistent matrix, then $\mathrm{k}=1$, and in inconsistent cases there may be more than one nonzero pairs of eigenvalues. The first column of $\mathbf{U}$ represents the rows (the first dimension), and the first column of $\mathbf{V}$ represents the columns (the second dimension) of $\mathbf{L}$. The first and the second columns of $\mathbf{U}$ and $\mathbf{V}$ generate the first component $\mathbf{L}_{1}$ of the matrix $\mathbf{L}$. The second component $\mathbf{L}_{2}$ is formed by the third and fourth columns of $\mathbf{U}$ and $\mathbf{V}$, and so on. If there are $\mathrm{k}$ positive eigenvalues $\delta_{1}, \ldots, \delta_{k}$ of $\mathbf{L L}^{\prime}$, then there are $\mathrm{k}$ decompositions $\mathbf{L}_{1}, \ldots, \mathbf{L}_{\mathrm{k}}$ corresponding to these eigenvalues. The result is $\mathbf{L}=\mathbf{L}_{1}+\ldots+\mathbf{L}_{k}$ where the matrices $\mathbf{L}_{j}$ are skew symmetric. If we write $\mathbf{R}_{\mathrm{j}}=\exp \left(\mathbf{L}_{\mathrm{j}}\right)$, we get $\mathbf{R}=\mathbf{R}_{1} * \ldots *{ }^{*} \mathbf{R}_{\mathrm{k}}$, where ${ }^{*}$ means elementwise product of matrices. The matrices $\mathbf{R}_{\mathrm{j}}$ are positive reciprocal matrices. 
In the decomposition $\mathbf{L}=\mathbf{L}_{1}+\ldots .+\mathbf{L}_{\mathrm{k}}$ component $\mathbf{L}_{\mathbf{j}}$ explains $100 \times \delta_{j} / \sum \delta_{i}$ percent of the total sum of squares of the elements of matrix $\mathbf{L}$ (total sum of the squared distances). If $\mathbf{R}$ is consistent, then the first component explains $100 \%$, and in an inconsistent case the first component $\mathbf{L}_{1}$ generally explains the greatest part, more than $90 \%$, of the sum of squares. Nature of the inconsistency connected to the first component can be visualized by plotting a figure where the compared alternatives are presented in the $(\mathrm{U}, \mathrm{V})$-plane. The coordinates in the first dimension can be found in the first column of $\mathbf{U}$, and the coordinates in the second dimension establish the first column of $\mathbf{V}$. In the consistent case the points settle on a straight line, but in an inconsistent case the points form a nonlinear figure telling about the nature of the inconsistency. If the inconsistency has a complicated nature, then the second component $\mathbf{L}_{2}$ explains a considerable part, too, and the corresponding plot in the $(\mathrm{U}, \mathrm{V})$-plane reveals background of the inconsistency. There are also natural interpretations for the first and second dimensions in the $(\mathrm{U}, \mathrm{V})$-plane. The interpretations can be found by observing correlations between the coordinates and the Euclidean distances of the rows or the Euclidean distances of the columns in matrix $\mathbf{L}$.

The estimates for the AHP-weights of the alternatives are based on the first component of the decomposition. By using the coordinates in the $(\mathrm{U}, \mathrm{V})$-plane, a matrix of signed distances between the columns and another matrix of signed distances between the rows can be formed. The sum of these two matrices is a compromise of the comparisons between the comparisons in the column direction and in the row direction. This skew symmetric matrix, say $\mathbf{A}$, must be normalized by requiring that its trace $\left(\mathbf{A A}^{\prime}\right)$ is equal to the trace of matrix $\mathbf{L}_{\mathbf{1}} \mathbf{L}_{\mathbf{1}}$ '. By exponentiation, a positive reciprocal consistent comparison matrix is found, and the weights can be calculated by using the standard eigenvalue method. The advantage of this procedure is that there is no need to apply the eigenvalue method to an inconsistent matrix.

\section{REFERENCES}

[1] Gass, S.I. and Rapcsák, T., 2001, Singular value decomposition in AHP. Proceedings of the Sixth International Symposium on the Analytic Hierarchy Process, ISAHP 2001, Bern.

[2] Saaty, T.L., 2000. Fundamentals of Decision Making and Priority Theory with The Analytic Hierarchy Process. RWS Publications, Pittsburgh. 